\title{
Erratum to: Choosing Where and How to Work
}

Andrea Flory

\section{Erratum to:}

Chapter 5 in: K. Chretien (ed.), Mothers in Medicine, https://doi.org/10.1007/978-3-319-68028-6_5

The chapter was inadvertently published with author's personal email and postal address. This has now been removed by this erratum.

The updated online version of this chapter can be found at https://doi.org/10.1007/978-3-319-68028-6_5
A. Flory, MD ( $\triangle)$
Washington, DC, USA
e-mail: alflory@gmail.com 